\title{
Correction to: Melatonin prevents bone destruction in mice with retinoic acid-induced osteoporosis
}

Xudong Wang ${ }^{1 \dagger}$, Tongzhou Liang ${ }^{1 \dagger}$, Yuanxin Zhu ${ }^{1 \dagger}$, Jincheng Qiu' ${ }^{1}$, Xianjian Qiu ${ }^{1}$, Chengjie Lian², Bo Gao ${ }^{1}$, Yan Peng ${ }^{1}$, Anjing Liang ${ }^{1}$, Hang Zhou ${ }^{2}$, Xiaoming Yang ${ }^{2}$, Zhiheng Liao ${ }^{2}$, Yongyong Li ${ }^{3}$, Caixia Xu ${ }^{3}$, Peiqiang $\mathrm{Su}^{2^{*}}$ and Dongsheng Huang ${ }^{1 *}$ (D)

\section{Correction to: Mol Med (2019) 25:43}

https://doi.org/10.1186/s10020-019-0107-0

Following publication of the original article (Wang et al. 2019), the authors identified an error in Fig. 4. The correct figure is given in this Correction article.

The original article can be found online at https://doi.org/10.1186/s10020019-0107-0.

*Correspondence: supq@mail.sysu.edu.cn; hdongsh@mail.sysu.edu.cn ${ }^{\dagger}$ Xudong Wang, Tongzhou Liang and Yuanxin Zhu contributed equally to this work

${ }^{1}$ Department of Orthopedics, Sun Yat-Sen Memorial Hospital of Sun Yat-Sen University, \#107 West Yan Jiang Road, Guangzhou 510120, Guangdong, China

${ }^{2}$ Department of Orthopedics, The First Affiliated Hospital of Sun Yat-Sen

University, \#58 Zhongshan Road II, Guangzhou 510080, Guangdong,

China

Full list of author information is available at the end of the article 


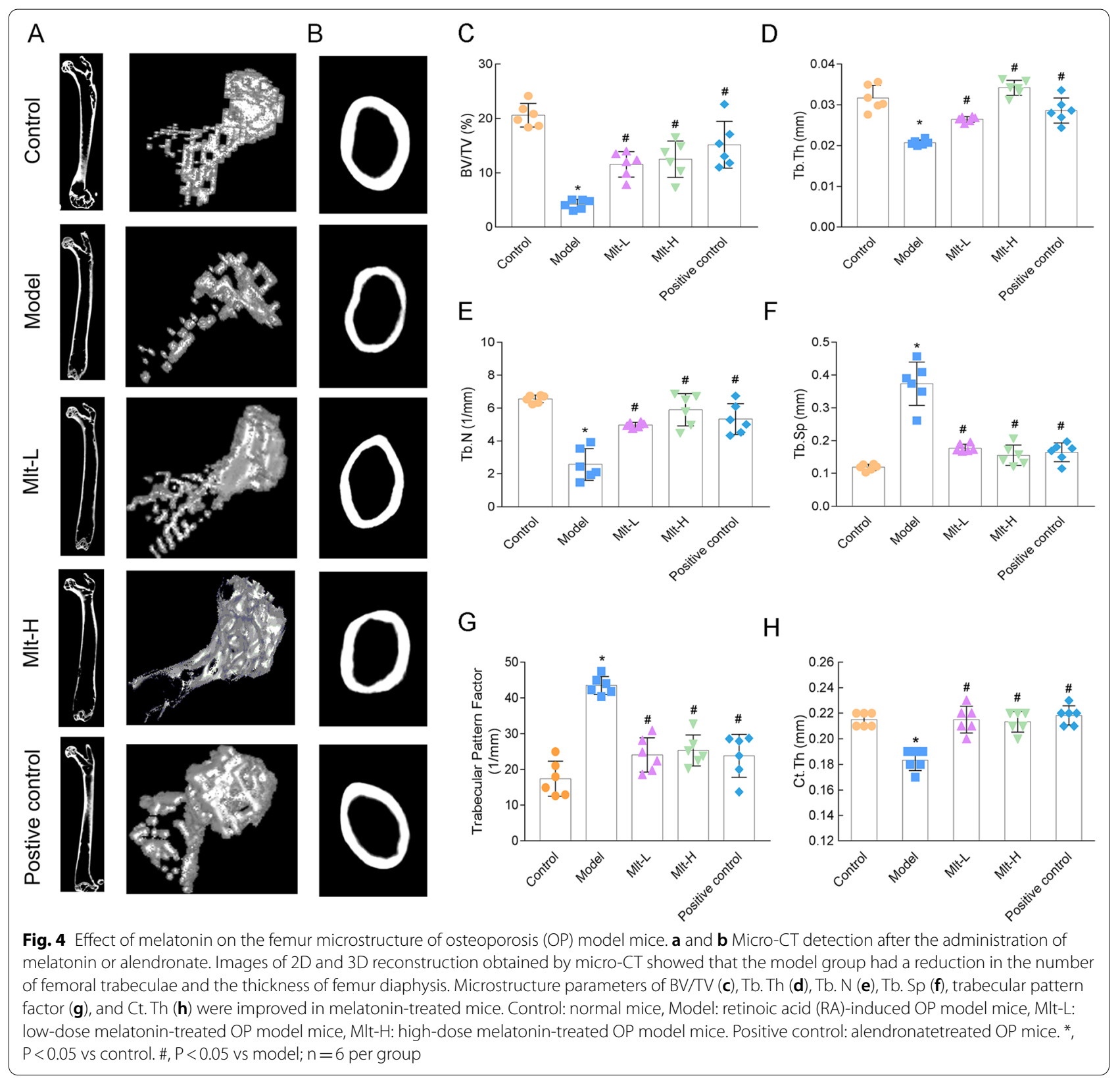

\section{Author details}

${ }^{1}$ Department of Orthopedics, Sun Yat-Sen Memorial Hospital of Sun Yat-Sen University, \#107 West Yan Jiang Road, Guangzhou 510120, Guangdong, China. ${ }^{2}$ Department of Orthopedics, The First Affiliated Hospital of Sun Yat-Sen University, \#58 Zhongshan Road II, Guangzhou 510080, Guangdong, China. ${ }^{3}$ Research Centre for Translational Medicine, The First Affiliated Hospital of Sun Yat-Sen University, Guangzhou 510080, Guangdong, China.

Published online: 26 October 2021

\section{Reference}

Wang X, Liang T, Zhu Y, Qiu J, Qiu X, Lian C, Gao Bo, Peng Y, Liang A, Zhou H, Yang X, Liao Z, Li Y, Caixia Xu, Peiqiang Su, Huang D. Melatonin prevents bone destruction in mice with retinoic acid-induced osteoporosis. Mol Med. 2019;25:43. https://doi.org/10.1186/s10020-019-0107-0.

\section{Publisher's Note}

Springer Nature remains neutral with regard to jurisdictional claims in published maps and institutional affiliations. 